\title{
Review of Beauty Production at HERA and Elsewhere
}

\author{
A.Geiser
}

DESY Hamburg, Germany

\begin{abstract}
Experimental results on beauty production at HERA are reviewed in the context of similar measurements at other colliders. As a result of a phenomenological study of the QCD scale dependence of many different NLO and NNLO predictions, a modification of the "default" scale choice is advocated. Experimental constraints on the photon-quark coupling are also investigated. [1]
\end{abstract}

\section{Introduction}

Beauty production at HERA (Fig. 1) is an important tool to investigate our present understanding of the theory of Quantum Chromo-Dynamics (QCD). On one hand, the large $b$ quark mass, taken as a hard scale, ensures that the cross sections are always perturbatively calculable. On the other hand, the simultaneous presence of competing hard scales, such as the transverse momentum $\left(p_{T}\right)$ of the heavy quark, or the virtuality of the exchanged photon $\left(Q^{2}\right)$, induces additional theoretical uncertainties due to terms in the perturbative expansion which depend logarithmically on the ratio of these scales. The comparison of the measured cross sections with theory predictions is therefore particularly sensitive to the way the perturbative expansion is made, and can therefore potentially discriminate how adequate a particular QCD scheme is for the decription of the cross section in question. This can also yield insights for other QCD processes at HERA, and for related

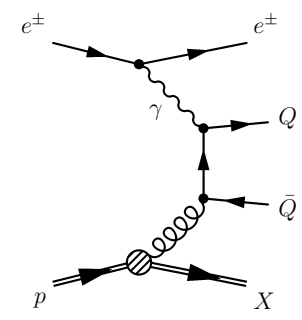

Figure 1: Feynman graph for the production of a heavy quark pair via the boson-gluonfusion (BGF) process. processes at other colliders, including future measurements at the LHC.

Since beauty in Deep Inelastic Scattering (DIS) is covered elsewhere [2], this contribution will concentrate on the photoproduction case $\left(Q^{2}<1 \mathrm{GeV}^{2}\right)$, in which the photon is quasireal. For beauty photoproduction at HERA, possible theoretical schemes include

- The leading order plus parton shower approach, where leading order (LO) QCD matrix elements are complemented by parton showers, usually using the DGLAP [3] parton evolution equations. This approach is implemented in many Monte Carlo models, and mostly used for the purpose of acceptance corrections.

- The kt-factorization approach [4], which can alternatively be used for parton showering, combined with the use of generalized parton density functions.

- The next-to-leading order (NLO) massive approach [5]. In this approach, the heavy quark mass is fully accounted for, and heavy quarks are therefore always produced dynamically in the matrix element, as illustrated by Fig. 1. Alternative LO processes, such as flavour excitation in the photon or the proton, are treated as next-to-leading order corrections to this BGF process. Processes in which the photon acts as a hadron-like source of light quarks or gluons are also included, but make only a small contribution. This approach is expected to work best when all relevant hard scales, e.g. $p_{T}$, are of order $m_{b}$.

- For $p_{T} \gg m_{b}$, large $\log p_{T} / m_{b}$ terms could in principle spoil the reliability of the predictions. In this case, it might be preferable to switch to a so-called massless scheme, in 
which the $b$ mass is neglected kinematically. The potentially large logarithms can then be resummed to all orders (next-to leading log or NLL resummation). Since such an approach is obviously not applicable when $p_{T} \sim m_{b}$, schemes have been designed which make a continous transition between the fixed order (FO) massive, and the NLL massless scheme. This is often referred to as the FONLL scheme [6].

On the experimental side, several different methods are used to tag the beauty final state. The $b$ quark can decay semileptonically into a muon or electron, which can be identified in the detector. The large momentum of the lepton transverse to the direction of the $b$-initiated jet, due to the sizeable $b$ mass, can be used to discriminate against semileptonic charm decays or misidentified light flavour events. The finite lifetime of the $B$ hadrons can lead to a measureable offset of the decay vertex with respect to the primary vertex of the event, which also leads to a significant impact parameter of the resulting secondary tracks. Finally, a lepton

HERA

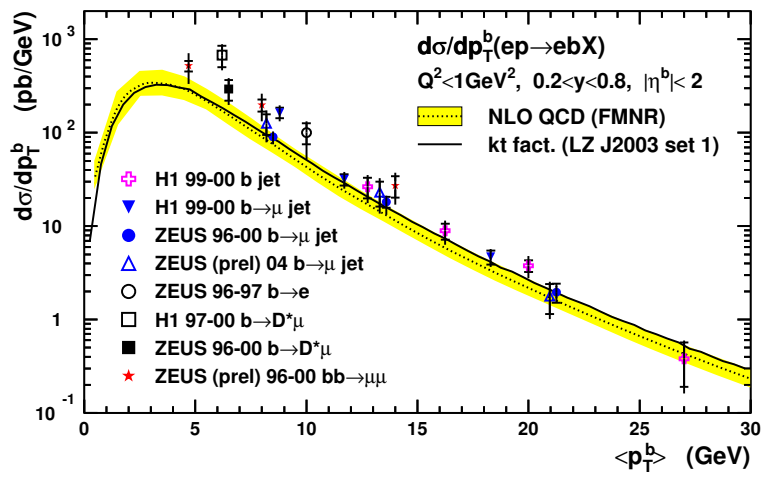

Figure 2: Beauty production cross section measurements in photoproduction at HERA as function of the transverse momentum of the $b$ quark, compared to QCD predictions. tag can e.g. be combined with a lifetime tag, with a second lepton tag, or with a $D^{*}$ meson from a $b$ decay.

Fig. 2 shows a compilation of all recent HERA measurements of $b$ photoproduction [7]. Reasonable agreement is found with both the fixed order NLO QCD prediction [5], and with a prediction based on kt factorization [8]. However, the data tend to lie somewhat above the central prediction in both cases.

A longstanding apparent discrepancy between data and theory in $b$ production at the Tevatron was resolved by combining a more careful consideration of $B$ fragmentation and decay parameters with an FONLL-based prediction [9]. This raises the question whether an FONLL predicton, which does not yet exist for $b$ production at HERA, would yield an improved agreement.

For this purpose, consider $b$ production at the $S p \bar{p} S$ collider, which had an effective partonparton center-of-mass energy very similar to that of HERA. Fig. 3 shows the measured $b$ quark

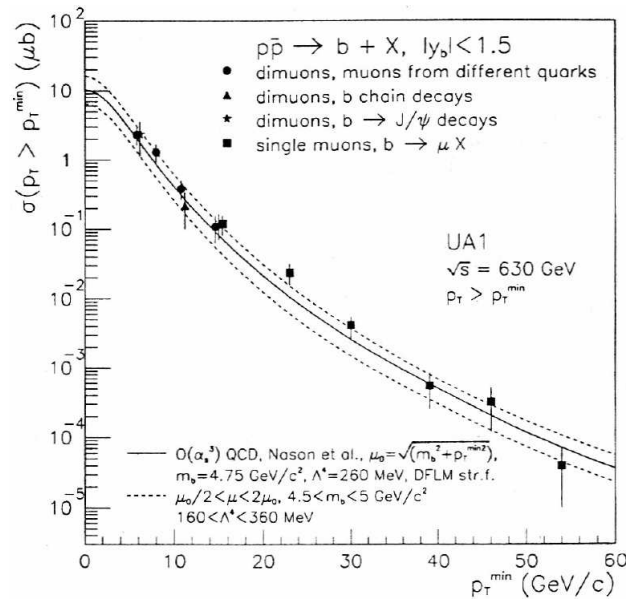

Figure 3: Integrated beauty production cross section at the $S p \bar{p} S$ as function of the minimum $p_{T}$ of the $b$ quark, compared to NLO QCD predictions. cross section [10] compared to the original NLO calculation [11]. Good agreement was observed at a time when the Tevatron experiments were starting to claim a discrepancy. Fig. 4 shows the same original data [10] compared to the more recent FONLL calculation at $b$ quark and $B$ hadron level $[9,12]$, with identical 
parameters as those used for the Tevatron. Good agreement is observed, also at muon level [12], even though NLO predictions at $B$ hadron and muon level were not available when the measurements were made. This indicates that the $B$ fragmentation and decay spectra, which had been studied carefully $[10,13]$, were treated consistently in these measurements. Furthermore, the NLO and FONLL predictions agree very well with each other, indicating that the large logs mentioned above do not yet play a significant role in this $p_{T}$ range (similar to the one at HERA). This can also be seen in charm production at HERA [14] for which an FONLL prediction exists.
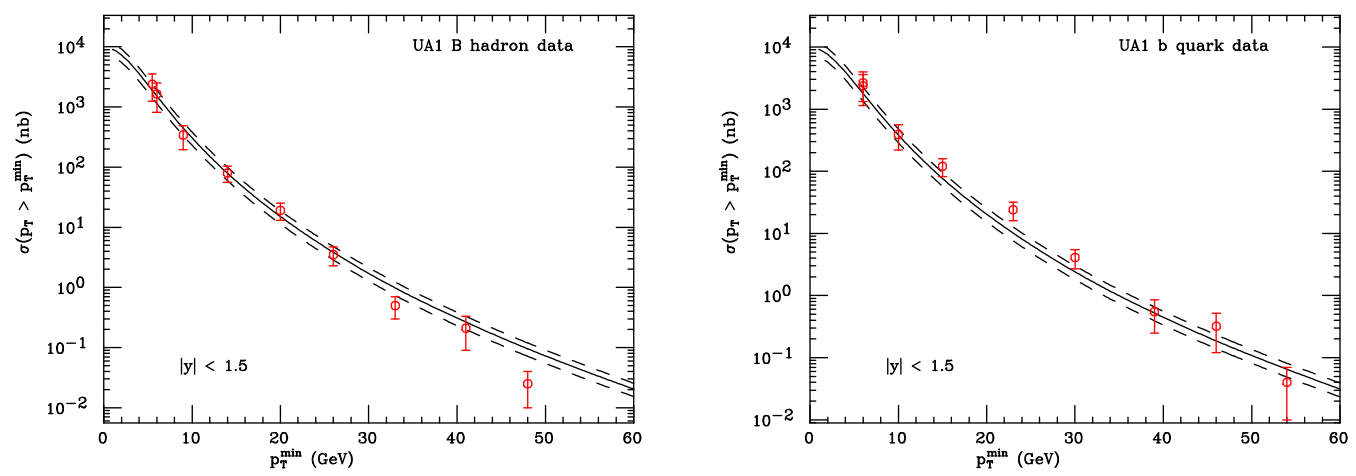

Figure 4: Integrated beauty production cross section at the $S p \bar{p} S$ as function of the minimum $p_{T}$ of the $B$ hadron (left) and $b$ quark (right), compared to preliminary FONLL QCD predictions [12].

In conclusion, an FONLL prediction for beauty production at HERA would be useful, but is not expected to significantly alter the data/theory comparison.

The dominant contribution to the theoretical error band of Fig. 1 is the variation of the renormalization/factorization scale by a factor 2 around the default scale $\mu_{0}=\sqrt{m_{b}^{2}+p_{T}^{2}}$. Such a variation is intended to reflect the uncertainty due to uncalculated higher orders. It might therefore be useful to reconsider this scale choice.

Ideally, in a QCD calculation to all orders, the result of the perturbatve expansion does not depend on the choice of these scales ${ }^{\mathrm{a}}$. In practice, a dependence arises from the truncation of the perturbative series. Since this is an artefact of the truncation, rather than a physical effect, the optimal scale can not be "measured" from the data. It must thus be obtained phenomenologically.

Traditionally, there have been several options to choose the "optimal" scale, e.g.

- The "natural" scale of the process. This is usually taken to be the transverse energy $\left(E_{T}\right)$ of the jet for jet measurements, the mass $m$ of a heavy particle for the total production cross section of this particle, or the combination $\sqrt{m^{2}+p_{T}^{2}}$ for differential cross sections of such a particle. Often, this is the only option considered. The choice of this natural scale is based on common sense, and on the hope that this will minimize the occurrance of large logs of the kind described above, for the central hard process. However, higher order subprocesses such as additional gluon radiation often occur at significantly smaller scales, such that this choice might not always be optimal.

${ }^{\mathrm{a}} \mathrm{As}$ is common practice, we will not distinguish between the factorization and renormalization scales in the following, and set both to be equal. A separate optimization of the two scales, which should be done in principle, will be left for future consideration. 
- The principle of fastest apparent convergence (FAC) [15]. The only way to reliably evaluate uncalculated higher orders is to actually do the higher order calculation. Unfortunately, this is often not possible. Instead, one could hope that a scale choice which makes the leading order prediction identical to the next-to-leading order one would also minimize the NNLO corrections. Since it can not be proven, this principle, which can be found in many QCD textbooks, has not been used very much recently. However, recent actual NNLO calculations might indicate that it works phenomenologically after all (see below).

- The principle of minimal sensitivity (PMS) [16]. The idea is that when the derivative of the cross section with respect to the NLO scale variation vanishes, the NNLO crrections will presumably also be small. Again, there is no proof that this textbook principle should work, but actual NNLO calculations might indicate that it does (see below).

To illustrate these principles, consider two examples. First, the prediction for the total cross section for beauty production at HERA-B [17] (Fig. 5). The natural scale for this case is the $b$ quark mass, $\mu_{0}=m_{b}$, and all scales are expressed as a fraction of this reference scale. Inspecting Fig. 5, one finds that both the PMS and FAC principles, applied to the NLO prediction and to the comparison with LO (NLO stability), would yield an optimal scale of about half the natural scale. The same conclusion would be obtained by using the $\mathrm{NLO}+\mathrm{NLL}$ prediction, including resummation, and comparing it to either the LO or the NLO prediction (NLO+NLL stability).
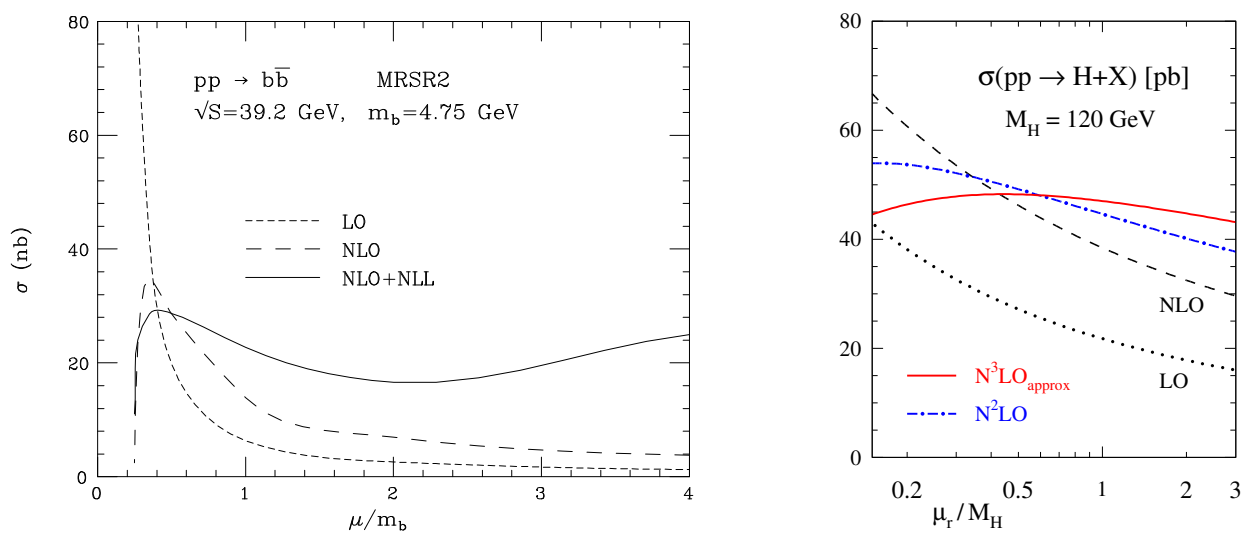

Figure 5: Scale dependence of the total cross section for beauty production at HERA-B [17] (left) and for Higgs production at LHC [18] for two different masses (right).

Second, the prediction for Higgs production at the LHC [18] (Fig. 5). The reference scale is now the Higgs mass $\left(\mu_{0}=m_{H}\right)$. However, inspecting the behaviour of the LO and NLO predictions, neither the FAC nor the PMS principle would yield a useful result in this case, since the two predictions do not cross, and the NLO prediction does not have a maximaum or minimum. This situation occurs rather frequently, and is also true for $b$ production at HERA. Fortunately, in the case of Higgs production, the NNLO and even NNNLO predictions have actually been calculated (Fig 5). Applying the FAC and PMS prescriptions to these instead (NNLO stability), again a scale significantly lower than the default scale would be favoured. This might indicate that choosing a scale which is smaller than the default one makes sense even if the FAC and PMS principles do not yield useful values at NLO. 
Beyond these examples, a more general study is needed to phenomenologically validate this approach. To avoid additional complications arising from a multiple scale problem caused by e.g. the scale $Q^{2}$ at HERA or the scale $M_{Z}$ at LEP, the study was limited to cross sections for photoproduction at HERA, or hadroproduction at fixed target energies, the Tevatron, and LHC. The somewhat arbitrary selection of processes includes beauty production at the $S p \bar{p} S[19,20]$, the Tevatron [20], and HERA-B [17], top production at the Tevatron [17, 20], direct photon production at fixed target [21], Z [22] and Higgs [18] production at the LHC, jets at HERA [23] and at the Tevatron [24]. This selection is obviously not complete. However, it is not biased in the sense that all processes that were considered were included, and none were discarded.

In each case the natural scale as defined above was used as a reference. In addition, wherever possible, the optimal scales from both the FAC and PMS principles, evaluated at NLO (NLO stability), NLO+NLL (NLO+NLL stability), and/or NNLO/NNNLO (NNLO stability) were evaluated separately. Fig. 6 shows the result of this evaluation. Each crossing point, maximum, or minimum in Fig. 5 yields one entry into this figure, and similarly for all the other processes. The conclusion is that the FAC and PMS principles tend to favour scales which are around $25-60 \%$ of the natural scale. Amazingly, this seems to be independent of whether these principles are applied at NLO, NLO+NLL, or NNLO level. For the jet [24] or b-jet [25] cross sections at the Tevatron, it has in part already become customary to use half the natural scale

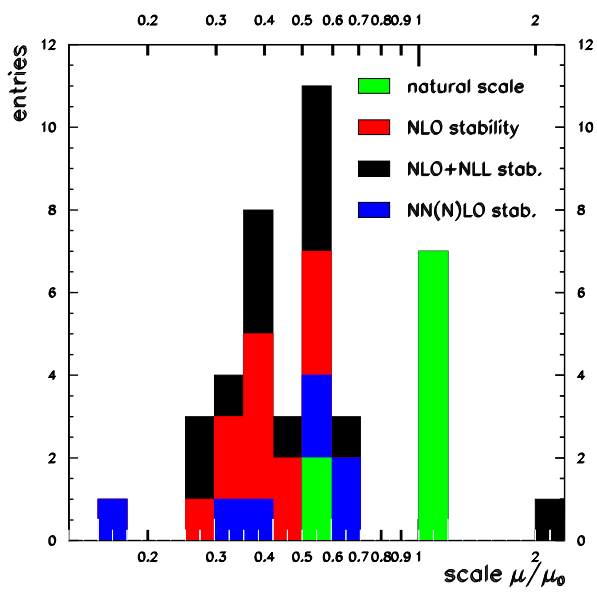

Figure 6: Summary of optimized scales derived as described in the text. as the central scale.

Using the natural scale as the default and varying it by a factor two, which is the choice adopted for most data/theory comparisons, covers only about half the entries, while the other half lies entirely below this range. Instead, using half the natural scale as the default and varying it by a factor two, thus still including the natural scale in the variation, covers about $95 \%$ of all the entries.

This yields the following conclusions.

- Obviously, whenever an NNLO calculation is available, it should be used.

- Whenever possible, a dedicated scale study should be made for each process for the kinematic range in question. Although there is no proof that the FAC and PMS principles should work, in practice they seem to give self-consistent and almost universal answers for processes at fixed target energies, HERA, the Tevatron, and the LHC.

- In the absence of either of the above, the default scale should be chosen to be half the natural scale, rather than the natural scale, in particular before claiming a discrepancy between data and theory. Empirically, this should enhance the chance that the NNLO calculation, when it becomes available, will actually lie within the quoted error band.

Now consider the application of the last proposal to actual data/theory comparisons. 
Fig. 7 shows the resulting comparison for beauty production at HERA. Although before the change of the default scale the agreement was already quite reasonable, the new choice, based on theoretical/phenomenological arguments, improves the agreement. A similar statement [1] can qualitatively be made for beauty production at the $S p \bar{p} S$ [10], the Tevatron [26], and even at RHIC [27], where a discrepancy has been claimed. The agreement with charm production at HERA [14] as well as charm [28] and top [29] production at the Tevatron also improves, or at least does not get worse. The same is true for inclusive jets at HERA, both in DIS [30] and in photoproduction [23]. In one case [31] half the scale has already been used in a published HERA result.

So far, no example is known to the author where the proposed change of default scale would result in a significant worsening of the data/theory agreement in a photo- or hadroproduction cross section. Thus, the phenomenologically motivated change seems to be supported by the data. It should therefore be considered to make it the default for future predictions at HERA, the Tevatron, and the LHC.

The investigations of the photon quark coupling are reported elsewhere [1].

\section{References}

[1] Slides: http://indico.cern.ch/materialDisplay.py?contribId=234\&sessionId=5\&materialId=slides\&confId=9499

[2] B. Kahle, these proceedings.

[3] V.N. Gribov and L.N. Lipatov, Yad. Fiz. 15 (1972) 781; L.N. Lipatov, Sov. J. Nucl. Phys. 20 (1975) 94; G.Altarelli, G. Parisi, Nucl. Phys. B126 (1977) 298; Y.L. Dokshitzer, Sov. Phys. JETP 46 (1977) 641.

[4] M. Ciafaloni, Nucl. Phys. B 296 (1988) 49;

S. Catani, F. Fiorani, and G, Marchesini, Phys. Lett. B234 (1990) 339;

S. Catani, F. Fiorani, and G. Marchesini, Nucl. Phys. B336 (1990) 18;

G. Marchesini, Nucl. Phys. B 445 (1995) 49.

[5] S. Frixione, P. Nason and G. Ridolfi, Nucl. Phys. B 454 (1995) 3 [hep-ph/9506226].

[6] M. Cacciari, S. Frixione and P. Nason, JHEP 0103 (2001) 006 [hep-ph/0102134].

[7] J. Breitweg et al. [ZEUS Collaboration], Eur. Phys. J. C 18 (2001) 625 [hep-ex/0011081];

S. Chekanov et al. [ZEUS Collaboration], Phys. Rev. D 70 (2004) 012008 [hep-ex/0312057];

A. Aktas et al. [H1 Collaboration], Eur. Phys. J. C 41 (2005) 453 [hep-ex/0502010];

A. Aktas et al. [H1 Collaboration], Phys. Lett. B 621 (2005) 56 [hep-ex/0503038];

S. Chekanov et al. [ZEUS Collaboration], Eur. Phys. J. C 50 (2007) 299 [hep-ex/0609050];

A. Aktas et al. [H1 Collaboration], Eur. Phys. J. C 47 (2006) 597 [hep-ex/0605016]; ZEUS Collaboration, contributed paper 269, XXII International Symposium on Lepton-Photon Interactions at High Energy, Uppsala, Sweden, 2005.

[8] A.V. Lipatov, N.P. Zotov Phys. Rev. D73 (2006) 114018 [hep-ph/0601240].

[9] M. Cacciari, P. Nason, Phys. Rev. Lett. 89 (2002) 122003 [hep-ph/0204025]. 
[10] C. Albajar et al. [UA1 Collaboration] Z. Phys. C 61 (1994) 41;

C. Albajar et al. [UA1 Collaboration] Phys. Lett. B 256 (1991) 121;

Erratum in Phys. Lett. B 262 (1991) 497.

[11] M.L. Mangano, P. Nason, and G. Ridolfi, Nucl. Phys. B373 (1993) 295.

[12] M. Cacciari, private communication.

[13] A. Geiser, PhD thesis, Aachen, Germany, PITHA 92/31, Oct 1992.

[14] J. Baines et al., Heavy quarks (Working Group 3): Summary Report for the HERA-LHC Workshop Proceedings, hep-ph/0601164.

[15] see e.g. G. Grunberg, Phys. Lett. B 95 (1980) 70.

[16] P. M. Stevenson, Phys. Rev. D23 (1981) 2916.

[17] R. Bonciani et al., Nucl. Phys. B529 (1998) 424 [hep-ph/9801375].

[18] S. Moch and A. Vogt, Phys. Lett. B 631 (2005) 48 [hep-ph/0508265].

[19] C. Albajar et al. [UA1 Collaboration], Phys. Lett. B369(1996) 46.

[20] S. Frixione et al., Adv. Ser. Direct. High Energy Phys. 15 (1998) 609 [hep-ph/9702287].

[21] S. Catani et al., JHEP 9903 (1999) 025 [hep-ph/9903436]

[22] C. Anastasiou et al., Phys. Rev. D 69 (2004) 094008 [hep-ph/0312266].

[23] S. Chekanov et al. [ZEUS Collaboration], DESY-07-092 [hep-ex/0706.3809]

[24] A. Abulencia et al. [CDF Collaboration], Phys. Rev. D75 (2007) 092006.

[25] S. Vallecorsa [CDF Collaboration], these proceedings.

[26] B. Reisert [CDF Collaboration], these proceedings.

[27] A. Mischke [STAR Collaboration], these proceedings.

[28] M. Bishai [CDF Collaboration] Phys. Rev. Lett. 91 (2003) 241804.

[29] CDF Collaboration, Preliminary Conf. Note 8148.

[30] A. Aktas et al. [H1 Collaboration], Eur. Phys. J. C46 (2006) 27.

[31] S. Chekanov et al. [ZEUS Collaboration] Eur. Phys. J. C44 (2005) 183 [hep-ex/0502007]. 